\title{
Multichannel Biphasic Muscle Stimulation System for Post Stroke Rehabilitation
}

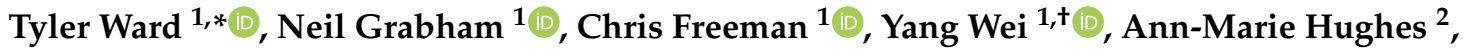 \\ Conor Power ${ }^{2}$, John Tudor ${ }^{1}$ and Kai Yang ${ }^{1(D)}$ \\ 1 School of Electronics and Computer Science, Faculty of Engineering and Physical Sciences, \\ University of Southampton, Southampton SO17 1BJ, UK; njg@ecs.soton.ac.uk (N.G.); \\ cf@ecs.soton.ac.uk (C.F.); yang.wei@ntu.ac.uk (Y.W.); mjt@ecs.soton.ac.uk (J.T.); ky2e09@soton.ac.uk (K.Y.) \\ 2 Health Sciences, Faculty of the Environment and Life Sciences, University of Southampton, \\ Southampton SO17 1BJ, UK; A.Hughes@soton.ac.uk (A.-M.H.); conor.power@soton.ac.uk (C.P.) \\ * Correspondence: t.ward@soton.ac.uk \\ + Current address: Department of Engineering at Nottingham Trent University, Nottingham NG1 4FQ, UK.
}

Received: 30 June 2020; Accepted: 14 July 2020; Published: 17 July 2020

\begin{abstract}
We present biphasic stimulator electronics developed for a wearable functional electrical stimulation system. The reported stimulator electronics consist of a twenty four channel biphasic stimulator. The stimulator circuitry is physically smaller per channel and offers a greater degree of control over stimulation parameters than existing functional electrical stimulator systems. The design achieves this by using, off the shelf multichannel high voltage switch integrated circuits combined with discrete current limiting and dc blocking circuitry for the frontend, and field programmable gate array based logic to manage pulse timing. The system has been tested on both healthy adults and those with reduced upper limb function following a stroke. Initial testing on healthy users has shown the stimulator can reliably generate specific target gestures such as palm opening or pointing with an average accuracy of better than 4 degrees across all gestures. Tests on stroke survivors produced some movement but this was limited by the mechanical movement available in those users' hands.
\end{abstract}

Keywords: electrode array; functional electrical stimulation; rehabilitation

\section{Introduction}

Stroke is a major cause of disability in the world. In 2017 there were approximately 12 million strokes globally [1]; prevalence is increasing due to improved longevity and rising obesity-related lifestyle factors (e.g., hypertension). Approximately $40 \%$ of stroke survivors are left with some loss of arm function as a result of their stroke [2]. Repetitive task-oriented training has been shown to help recovery as part of a rehabilitation programme [3] and one of the most prevalent technologies used to assist in rehabilitation is Functional Electrical Stimulation (FES) [4]. This method uses pulses of electrical current passing through underlying nerves, thereby causing the user's muscles to contract and generate movement. By repeated use of FES to facilitate task practice, some users may regain functional movement [5] in the affected limbs.

The SMARTmove project [6], funded by the UK Medical Research Council (MRC), realised a new FES system which provided high selectivity stimulation of the muscles used in hand, wrist and arm movements. The wearable system created by the SMARTmove project is the first example of combining a fabric FES array, a non-contact feedback sensor, and a controller able to precisely adjust stimulation using dynamic models of the arm. Although this wearable system is specifically designed for stroke survivors, who often exhibit movement dysfunction in one or more affected limbs, it can also be used 
in the rehabilitation of other neurological conditions (e.g., spinal cord injuries, multiple sclerosis) to restore lost or impaired movement.

Current commercial FES systems primarily use two pairs of large electrodes (typically of the order of $5 \mathrm{~cm} \times 5 \mathrm{~cm}$ in size), giving the ability to drive two groups of muscles, but these are unable to produce selective movement such as fine finger-level gestures. A few commercial systems such as the Bioness-H200, are able to more selectively drive several groups of muscles allowing for more precise gestures; the Bioness- $\mathrm{H} 200$ can control five different muscles in the hand/wrist. Academic research developing transcutaneous multi-pad electrodes and arrays has been driven by the University of Belgrade [7], ETH Zurich [8], the Swiss Federal Institute of Technology Lausanne [9], Sheffield Hallam University [10], together with Fatronik-Tecnalia [11]. The layout of electrode arrays often assumes a generic pattern, and is typically used to allow the placement of a virtual electrode at any location within the array [12]. Such solutions, however, are only able to control a few of these points at a time using sequential stimulation; this limits the ability to produce complex gestures. Other systems employ a multi-pad electrode layout which is designed to match the geometry of underlying muscles, however, these require precise alignment. In both cases of electrode layout, electrodes are activated in an open-loop fashion, and do not receive real-time position feedback to correct the resulting motion.

Existing systems also have limitations in terms of the electrode construction which restricts usability. The first widely-used approach uses embroidered conductive thread resulting in a rough electrode surface which causes an uneven current distribution, leading to discomfort when the stimulation current is high. In addition, embroidered high density conductive areas such as electrodes are stiff. The second widely-used approach uses gel electrodes on plastic substrates which are not sufficiently flexible, breathable, or comfortable.

The SMARTmove project has created a new FES system to address the limitations of existing FES techniques. By using an array of small electrodes, rather than a pair of large electrodes, a high level of selectivity in the stimulated muscles has been achieved. Independent control of smaller electrodes also enables more individual muscles to be activated simultaneously, thus increasing the range of achieved gestures. By using real-time feedback and an iterative learning control approach, the system can help the user achieve precise movements while adapting to their physiology, rather than being limited to a set of fixed stimulation patterns. This allows the stimulation to be better tailored to the user's progress and condition and avoids the need for exact electrode positioning. The printed dry fabric electrode array also makes the system easier to don/doff and avoids issues that arise with gel electrodes, such as drying out, susceptibility to contamination, and a short life time (typically 1 to 2 weeks). The fabric electrode array is more flexible and comfortable. It is also washable and reusable, and therefore cost effective for long term use.

This paper focuses on the design of the stimulation electronics used in the SMARTmove system. A review of stimulation electronics output stages for FES has been carried out by Souza [13]. The applied stimulation can be grouped into two general categories: monophasic where the stimulation pulses only have one polarity; and biphasic where the stimulation pulses have both positive and negative polarities. The stimulator design reported in this paper uses biphasic stimulation as this reduces the potential for skin burns and tissue damage [14] and provides a wider range of stimulation profiles when compared to monophasic stimulation. Of the biphasic designs investigated, the majority use H-bridge drivers for controlling the phase of the stimulation. However, this requires a pair of electrodes for each stimulation channel. Other non H-bridge designs often also require control of both the source and return of each channel, such as that by Huerta [15]. Where a common return electrode is used, as is the case when using an array, each channel needs to be able to generate both positive and negative stimulation voltages rather than swapping the polarity of the electrodes, as typically occurs with simple electrode pairs.

Existing FES stimulator designs are also often based around discrete components rather than using an integrated package, thereby increasing the circuit board space required for each channel. 
Many existing designs also use an isolation transformer on the output to provide an isolation barrier; these transformers often have a significant footprint which leads to a large implemented size when there are multiple channels.

Using existing solutions for FES stimulation would result in a bulky and complex stimulator that would be unsuitable for wearable use. To overcome these limitations the developed design uses off the shelf multichannel high voltage switchIntegrated circuits (ICs) combined with discrete current limiting and dc blocking circuitry for the stimulation frontend. Pulse timing is controlled by Field Programmable Gate Array (FPGA) based logic which is in turn controlled by a microprocessor to manage communication with the software managing the stimulation profiles. These provide greater parameter control than previous designs with a low cost and a small size suitable for wearable deployment.

In this paper we discuss, in Section 2, the constituent parts and operation of the rehabilitation solution as a whole. The designed electronics that make up the multichannel biphasic stimulator is discussed in Section 3. Section 4 presents system use and testing on both healthy individuals and stroke survivors, illustrating use of the system to stimulate individual finger movements to produce a range of gestures. Finally, conclusions are provided.

\section{Complete System: Components and Operation}

The SMARTmove system is designed to allow stroke survivors to practice a series of commonly undertaken tasks at home with the assistance of electrical stimulation, as part of their rehabilitation programme. Currently the system supports three different gestures (see Figure 1): palm flattening, pointing and pinching. These gestures form the basis for functional activities such as closing a drawer or operating a light switch.

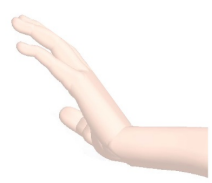

(a) palm

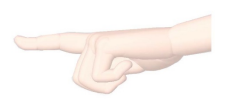

(b) point

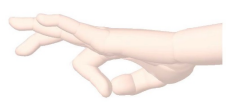

(c) pinch

Figure 1. Target gestures the system will assist the user in creating.

The SMARTmove system contains several components that work together to control and deliver the electrical stimulation. A graphical overview of the system components can be seen in Figure 2.

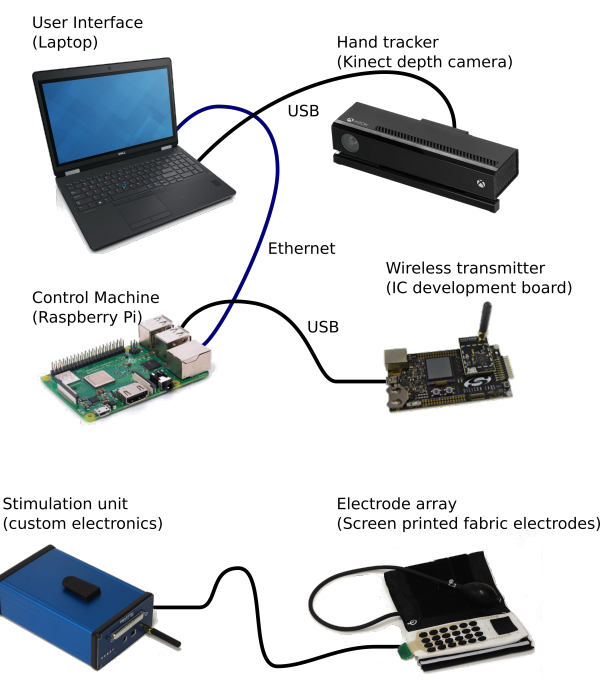

Figure 2. Overview of the SMARTmove system. 
The SMARTmove system uses a commercial, off the shelf, depth camera (Microsoft Kinect v2) to capture the movements of a user's hand. The depth information from the camera is processed on a laptop to extract the angular position of the user's hand and fingers. This processing is performed by a standalone application which then streams the data into the Graphical User Interface (GUI) application also running on the user's laptop.

The GUI provides the interface for the user to control the system. After initial configuration of the system a series of tasks of increasing complexity are presented to the user. The system uses a gamification approach to the rehabilitation process by enabling users to progress from task to task once they have gained enough points in a given task. These points are based on how well they perform a task using performance metrics, such as the accuracy of the final gesture and the smoothness of movement. This means that the user will progress onto the later tasks more quickly once they have become more proficient in the earlier tasks. The GUI application translates the user's interactions into a series of commands for the control software and displays the returned feedback information to the user after every task.

The control system [16] runs on a separate Raspberry Pi low-cost computer and receives the commands and hand tracking data from the GUI on the laptop. This is achieved using a network connection over a wired Ethernet link. The software has a map of which electrode causes which group of hand joint angles to move, and by how much. This map is automatically generated by monitoring the movement during an array identification sequence (described further in Section 4.1). An iterative learning controller is used in the control system, allowing it to improve the user's response by adjusting the delivered stimulation based on previous results. The stimulation levels which are calculated by the controller forty times a second are then sent over USB to the wireless transmitter which relays them wirelessly to the stimulator unit electronics (described in detail in Section 3).

The stimulation pulses are delivered to the user through a fabric electrode array [17], connected via a cable to the stimulation unit. This array consists of screen printed dry electrodes in a six by four pattern, giving twenty four stimulation electrodes in total, together with an additional large common return electrode. The electrode array is attached to a cuff which is placed on the arm and secured using a hook and loop fastener. The cuff applies pressure to the electrode array ensuring good contact between the electrodes and the user's skin.

\section{Stimulator Unit: Electronics Design and Implementation}

\subsection{Overview}

The stimulator unit converts the stimulation levels sent from the control software into the electrical stimulation pulses. Each set of levels from the controller triggers a single set of synchronised pulses from the unit. Each of the twenty four independent stimulation channels can have a different stimulation intensity level, including the possibility of zero stimulation. The stimulator uses voltage controlled stimulation in a multichannel configuration with varying pulse length used to adjust the stimulation intensity of each channel. Each channel has an independently controllable pulse width and waveform, adjustable on every pulse. The stimulation voltage is common across all the electrodes but is variable between $50 \mathrm{~V}$ and $100 \mathrm{~V} \mathrm{DC}$.

The design is implemented using a series of interconnected modules split across several custom printed circuit boards (PCBs). The modules are separated into a control board together with sufficient eight channel driver boards to produce the required number of stimulation channels. The setup for the SMARTmove system uses three driver boards to achieve twenty four channels. These boards are slotted inside a compact enclosure, shown in Figure 3, which can be worn, for example, on the user's belt. The boards are connected together with a series of short cable harnesses. The stimulator is connected to the fabric electrode array worn on the arm using a detachable multicore cable. Figure 4 is a schematic showing the functionality implemented on each board. 


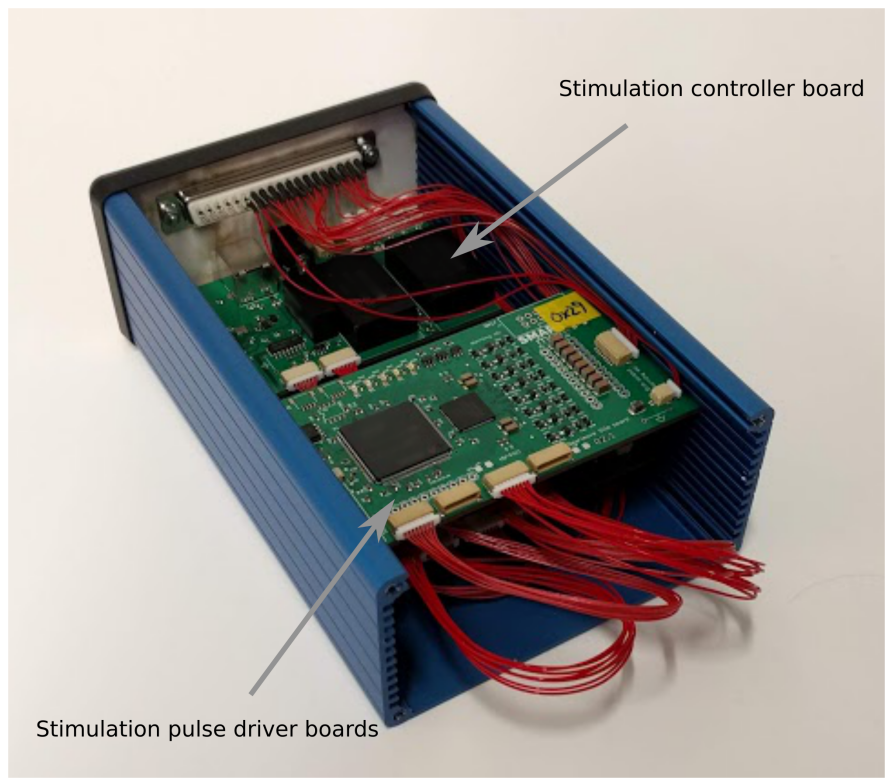

Figure 3. Interior of custom stimulation unit.

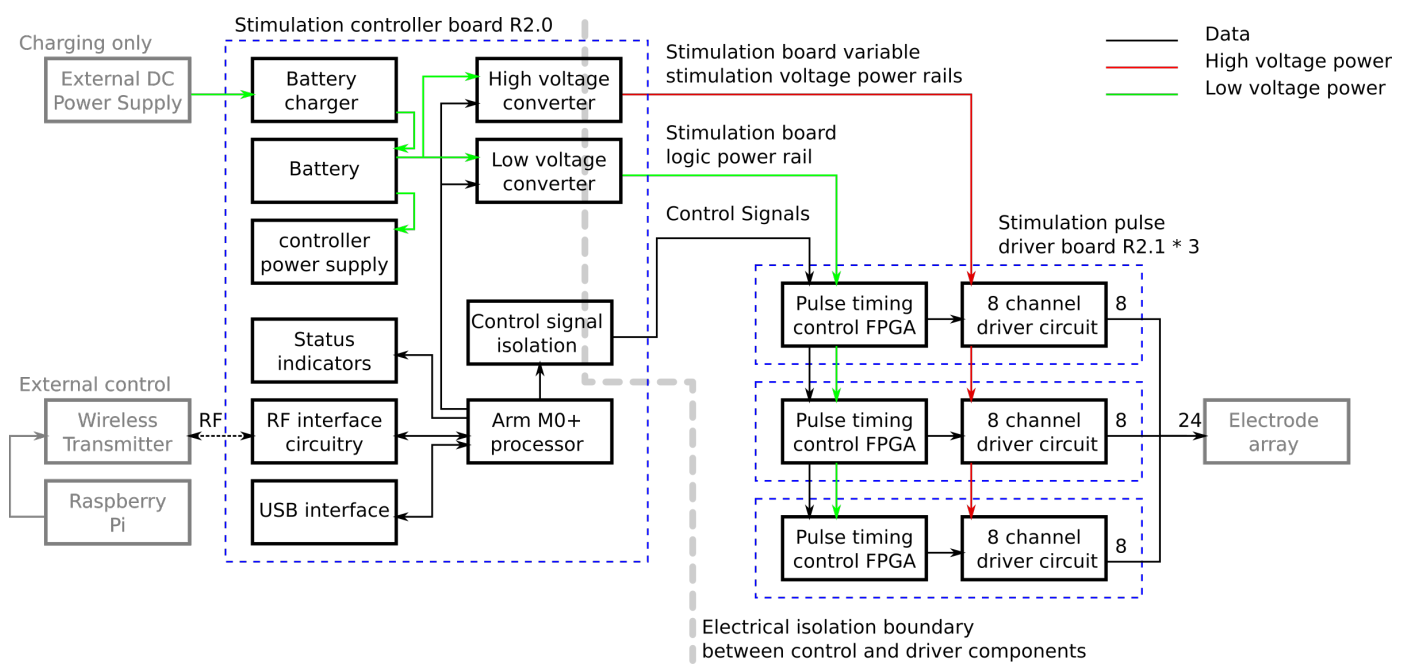

Figure 4. Block diagram of stimulator electronics.

\subsection{Stimulation Controller Board}

The controller board contains the modules for managing the system and passing commands from the control program to the driver boards. Commands are received from the control software running on the Raspberry Pi either over a USB cable or via a wireless link from a USB attached transmitter. Commands are processed by an ARM M0+ microprocessor which has inbuilt USB and wireless connectivity. Each stimulation request from the control program defines a single set of pulses, one for each channel, with forty stimulation requests being sent per second. Each set of pulses is defined separately which allows the length of every pulse to be varied, and prevents stimulation continuing should the connection to the Raspberry Pi be lost. When a message is received it is checked for corruption and, if it is valid, it is decoded into the pulse lengths for each channel and the voltages for the stimulation power supplies. The high voltage power supplies are reconfigured to provide a new stimulation voltage if it has changed. The pulse lengths for each channel are then sent to the corresponding driver over an $\mathrm{I}^{2} \mathrm{C}$ interface. In order to keep the pulses synchronised, a dedicated start signal is used which triggers all of the drivers to start the pulses at the same time. 
Another major function of the control board is management of the power supplies. To provide for wireless operation an internal battery is used to power the stimulator and all other required voltages are generated from this. The battery, which provides approximately four hours of active use, is charged using an external $9 \mathrm{~V}$ DC external power supply which can also be used to power the unit. Internal power regulators take either the battery voltage or DC input voltage and generate $3.3 \mathrm{~V}$ DC for the microprocessor and a switchable $5 \mathrm{~V} \mathrm{DC}$ supply for powering the rest of the system.

The stimulation voltage is generated using a pair of isolated variable DC-DC voltage converters which take the power from the $5 \mathrm{~V}$ power rail and produce between $50 \mathrm{~V}$ and $100 \mathrm{~V}$ DC which is selectable by the controller for each pulse. The DC-DC converters sit across an isolation boundary which separates the low voltage interface circuitry on the board from the high voltage stimulation circuitry located on the other boards. This boundary adds two safety features. In the event of major damage to the driver boards the high stimulation voltages cannot appear on any of the interface connectors. In addition, current cannot flow between the electrode interface and any of the other connectors on the unit. This means the stimulation current will only flow between the stimulation electrodes and the return electrode and will not take any unwanted paths through the user to an interface connector if touched. Power for the low voltage circuitry on the driver boards is generated by another isolated DC-DC converter at $5 \mathrm{~V}$ DC. Control signals for the driver boards are passed through optical isolation ICs in order to maintain the isolation barrier.

\subsection{Stimulation Pulse Driver Board}

The stimulation pulse's driver boards receive pulse length settings and triggering from the control board and produce the required pulses. Each pulse is made up of a variable length positive pulse followed by a variable length negative pulse. The electrode is then actively driven to $0 \mathrm{~V}$ after each pulse, where it remains until the next pulse.

The pulse drive circuitry, which generates the initial pulse waveform, consists of a constant voltage pulse generator which is capable of operating at high voltages. The pulse output is passed through a current limiting circuit and a DC blocking capacitor. The connections between the components can be seen in Figure 5.

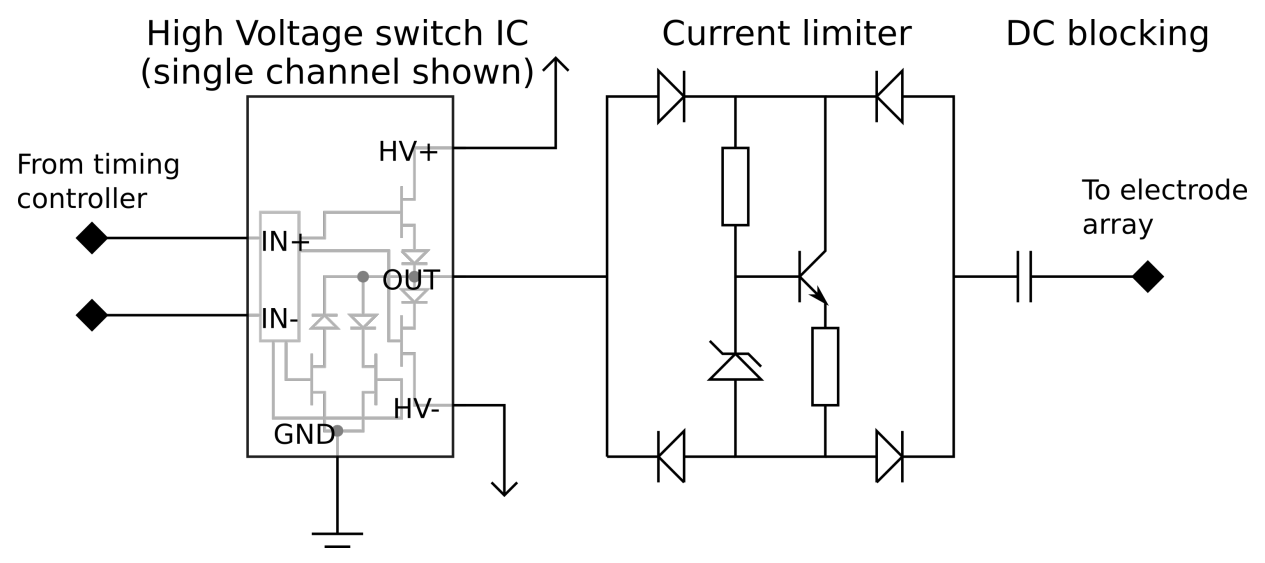

Figure 5. Circuit schematic of stimulation pulse driver board output stage.

The pulse generator uses a commercially available high voltage switch integrated circuit (IC). This IC contains a Field Effect Transistor (FET) based switch stage along with the circuitry to control the gates from logic level input signals. Each of the eight outputs can be driven to one of three states $(+\mathrm{V}, \mathrm{Gnd},-\mathrm{V})$ in any combination. This creates the positive and negative pulses as well as an active return to ground when not producing a pulse. Using an off the shelf IC provides a very compact implementation which fits in a $10 \mathrm{~mm}$ by $10 \mathrm{~mm}$ package.

In order to achieve user safety, protective circuitry is added to the output of each channel. Current limiting circuitry is placed after every output to prevent large stimulation currents from 
flowing if there is a low resistance path through the user. A Zener diode based current limiter is set to a $9 \mathrm{~mA}$ limit and placed within a diode bridge to limit the current in either direction. The final component in the pulse path is a DC blocking capacitor which prevents any DC voltage appearing on the electrodes which could potentially cause electrical burns in the event of hardware failure. These nine extra components per channel require minimal board space, each taking $80 \mathrm{~mm}^{2}$ of PCB surface area per channel. Once the driver IC and routing space to link the parts together are taken into account the required area is still very low; the eight channel driver along with the required extra circuitry will fit in an area of $20 \mathrm{~mm} \times 35 \mathrm{~mm}$ assuming a 4-layer PCB, of which $16 \mathrm{~mm} \times 20 \mathrm{~mm}$ comprises the extra safety circuitry. The integrated driver design also reduces the cost of the stimulator compared with using discrete control transistors for each channel.

The pulse generator is driven by a timing controller which controls the pulse lengths on each channel. The timing controller is implemented on a Field Programmable Gate Array (FPGA) which contains an output control block for each output and a communication module to provide an interface to the controller board. In the developed design each driver IC has its own control FPGA in order to allow the number of channels to be easily altered if required. In a less flexible hardware configuration a control FPGA could control multiple driver ICs to reduce cost/size. The output control block contains a high accuracy timer and state machine which cycles through the required stages of the pulse. The control blocks in the FPGA have several waveforms available allowing them to produce a range of output pulse waveforms as can be seen in Figure 6 . The default mode used in the majority of the tests to date was the biphasic negative mode which uses a matched pair of pulses where the negative pulse is immediately followed by a positive pulse as shown in Figure 7.
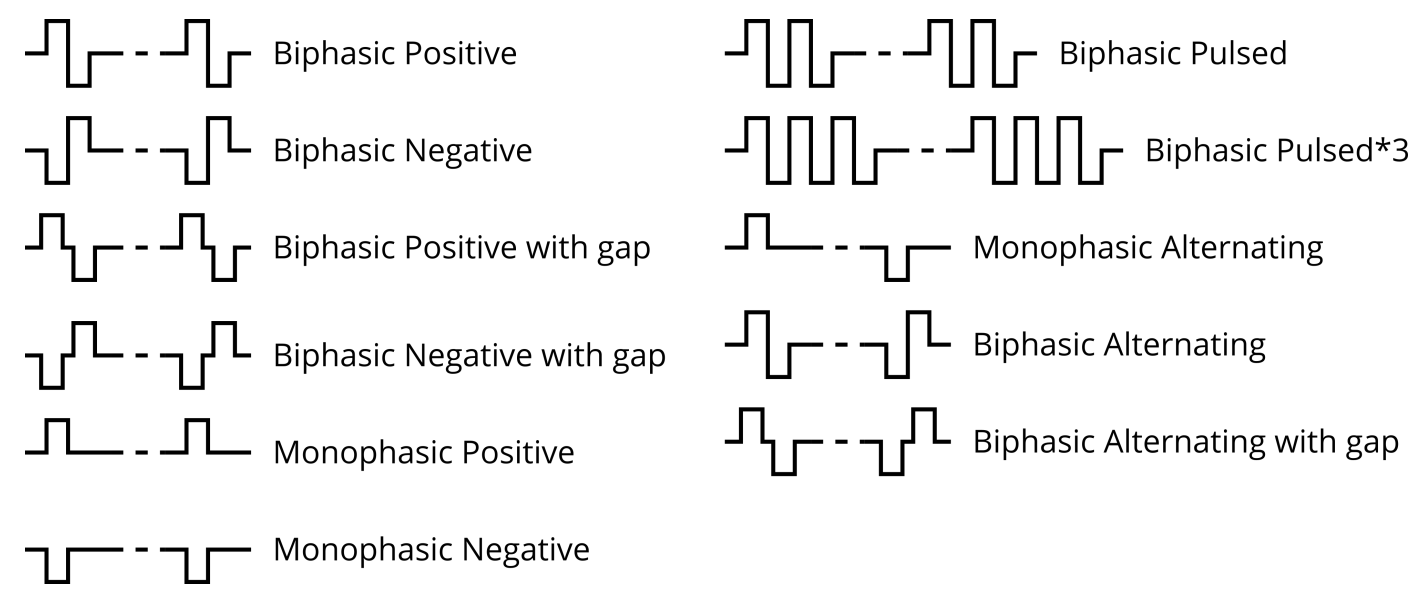

Figure 6. Stimulator output waveform options.

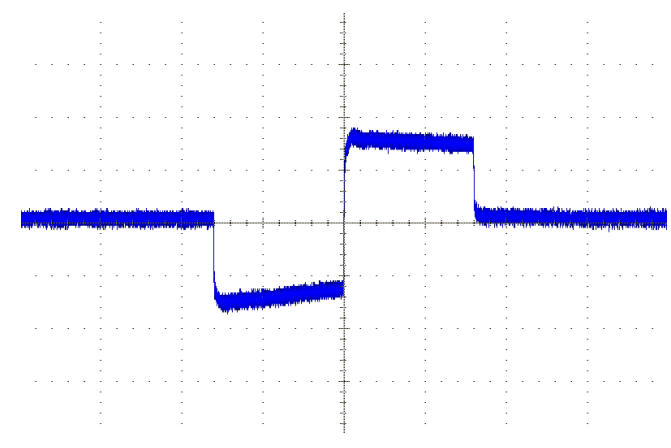

(a) Pulse Waveform without electrode connected to skin.

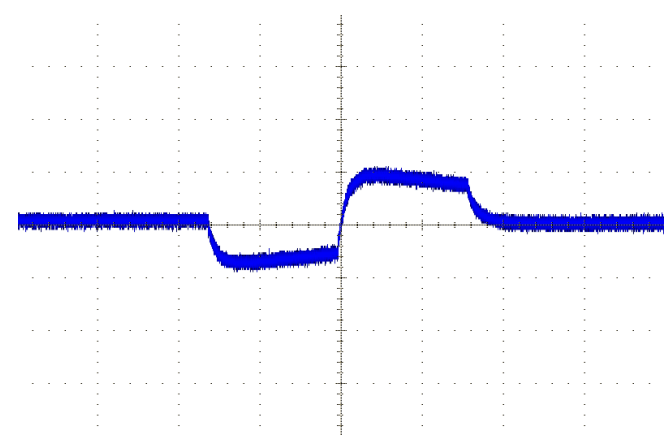

(b) Waveform with electrode connected to the skin showing the current limit in operation.

Figure 7. Stimulator pulse waveforms (oscilloscope settings $50 \mathrm{~V} / \mathrm{div}, 100 \mu \mathrm{s} / \mathrm{div}$ ). 
The controller board interface is in two parts. The pulse lengths are sent to the drivers using the isolated $\mathrm{I}^{2} \mathrm{C}$ interface on the control board. The controller addresses each of the driver boards in turn sending them the settings for each channel for the next pulse. The start trigger signal is then sent using a dedicated logic signal to all boards at once. This allows all the boards to trigger the output control blocks thus starting their outputs close to simultaneously. Differences in timing of up to 20 ns between the outputs of different driver boards are possible as the FPGA clocks are not synchronised but this is negligible compared to the typical pulse lengths of $60 \mu$ s to $500 \mu \mathrm{s}$. A simplified block diagram showing how these interface with the rest of the components in the timing controller can be found in Figure 8.

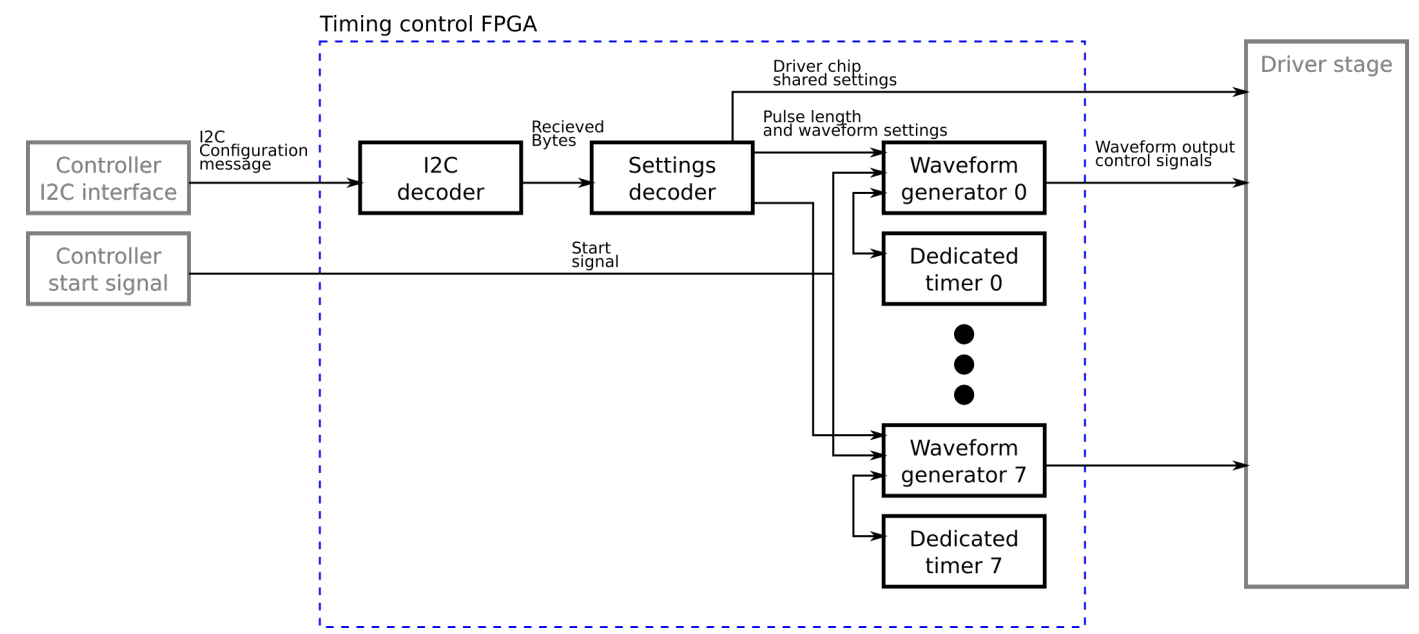

Figure 8. Simplified block diagram of Field Programmable Gate Array (FPGA) logic blocks used to generate pulse waveforms.

\section{System Testing}

\subsection{System Setup}

The cuff containing the electrode array is first placed on the arm and secured in place. Exact placement is not required as the control system will automatically learn and adjust the stimulation according to measured performance. The next stage sets the maximum stimulation level. This needs to be undertaken before first use, each time the system is used, as conditions such as humidity and skin hydration level can affect a user's response on any given day. Generally, higher stimulation levels result in more precise movements, however, with high stimulation levels discomfort can occur. The stimulation level is adjusted to the maximum level that can be tolerated without any discomfort for the user.

The next stage of the setup process is to perform an array identification sequence which applies stimulation to each electrode in turn and the system measures the resultant movement as the stimulation level is ramped up to the set maximum level. These measured movements are stored so that they can be used to calculate the stimulation required for each target gesture. This step need only be carried out during the first few instances of using the system. During these first few uses the control system will have recorded how the user responds under different conditions or differences in array placement. Then, in future use, the control system will observe the first few gestures and use recorded data to determine how to apply the stimulation.

The hand positions for each of the three target gestures and an initial hand position are then recorded. Healthy subjects create the target gestures themselves; those with impaired movement need another person to make the reference gesture for them. 


\subsection{System Use}

Once the setup is complete the system can stimulate the muscles to produce the required gestures. A target gesture is selected on the GUI application and the test is started. The system then calculates the required stimulation pattern to move from the initial position to the desired gesture, as can be seen in Figure 9. The stimulation is then delivered and the resultant motion is observed and fed back into the iterative learning control stimulation algorithm to improve the resulting gesture at the next stimulation run. The user will perform several stimulation runs for a gesture before advancing to the next gesture.

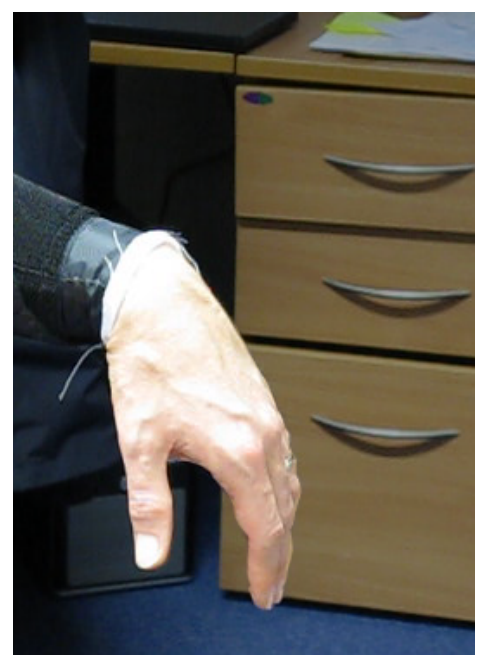

(a) Initial position (at rest)

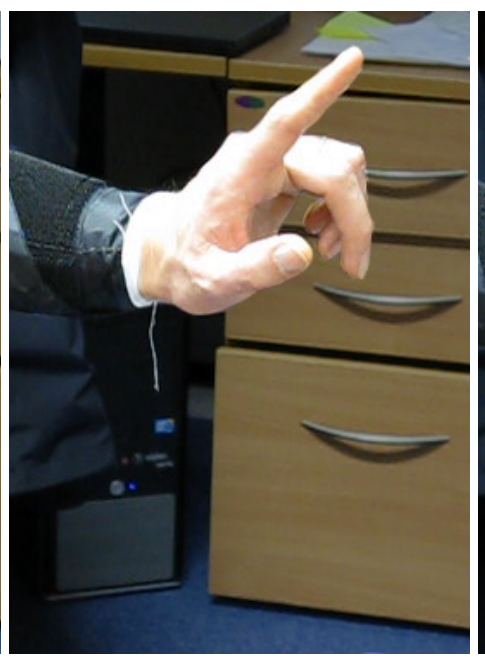

(b) Reference gesture (manually produced)

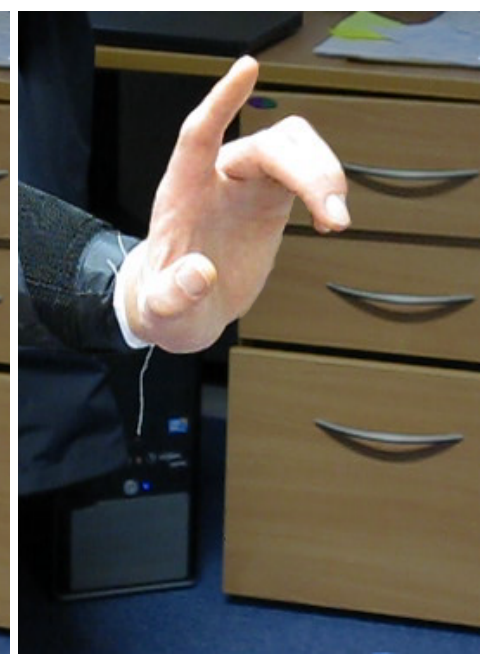

(c) Driven gesture (stimulator produced)

Figure 9. Photographs showing target and produced gestures.

\subsection{Testing on Healthy Volunteers and Stroke Survivors}

The stimulation electronics has been tested as part of the SMARTmove system on five healthy participants as well as three members of the End User Group (EUG) of stroke survivors.

\subsubsection{Initial Tests with Healthy Participants}

Initial tests were carried out on five healthy participants, following ethical approval. The required maximum stimulation level which was comfortable was found to differ significantly between users, with acceptable pulse lengths varying from $60 \mu \mathrm{s}$ to $500 \mu \mathrm{s}$. The system was able to produce a range of movements when testing individual electrodes, and the gesture tests were able to produce accurate gestures. The accuracy of the driven gestures was measured by the control software using angles received from the camera tracking data. The data was processed using three functions to produce values for comparison. These were also used to provide feedback to the user through the GUI.

Equation (1) was used to calculate the 'percent performance': this is a percentage score of the movement as a proportion of the total movement required to reach the target gesture. Equation (2) provided the 'mean error': this is the average absolute error between the produced gesture and the target gesture in degrees. Equation (3) provided the 'percent ROM performance': this is the performance relative to the range of movement (ROM), a high score here shows the muscles are being stimulated to move in the correct direction even if they do not achieve the necessary scale of movement required to fully produce the gesture. In these equations $\theta$ represents the vector of joint angles after stimulation, $\theta_{R E F}$ the vector of target angles, $\theta_{R O M}$ the joint angle ROM values, and $\theta_{I N I}$ the initial joint angles.

$$
100\left(1-\frac{\left\|\theta_{R E F}-\theta\right\|}{\left\|\theta_{R E F}-\theta_{I N I}\right\|}\right)
$$




$$
\begin{gathered}
\left\|\theta_{\text {REF }}-\theta\right\| \\
100\left(1-\frac{\left\|\theta_{\text {REF }}-\theta\right\|}{\left\|\theta_{\text {REF }}-\theta_{\text {ROM }}\right\|}\right) .
\end{gathered}
$$

Figure 10 shows a series of stimulation runs performed on one participant targeting the three different gestures shown in Figure 1. The mean error of the joint angles between the achieved position and the target position is shown in the centre. The left graph is the accuracy as a percentage of the total movement required, and the right graph shows the accuracy as a percentage of the range of movement.
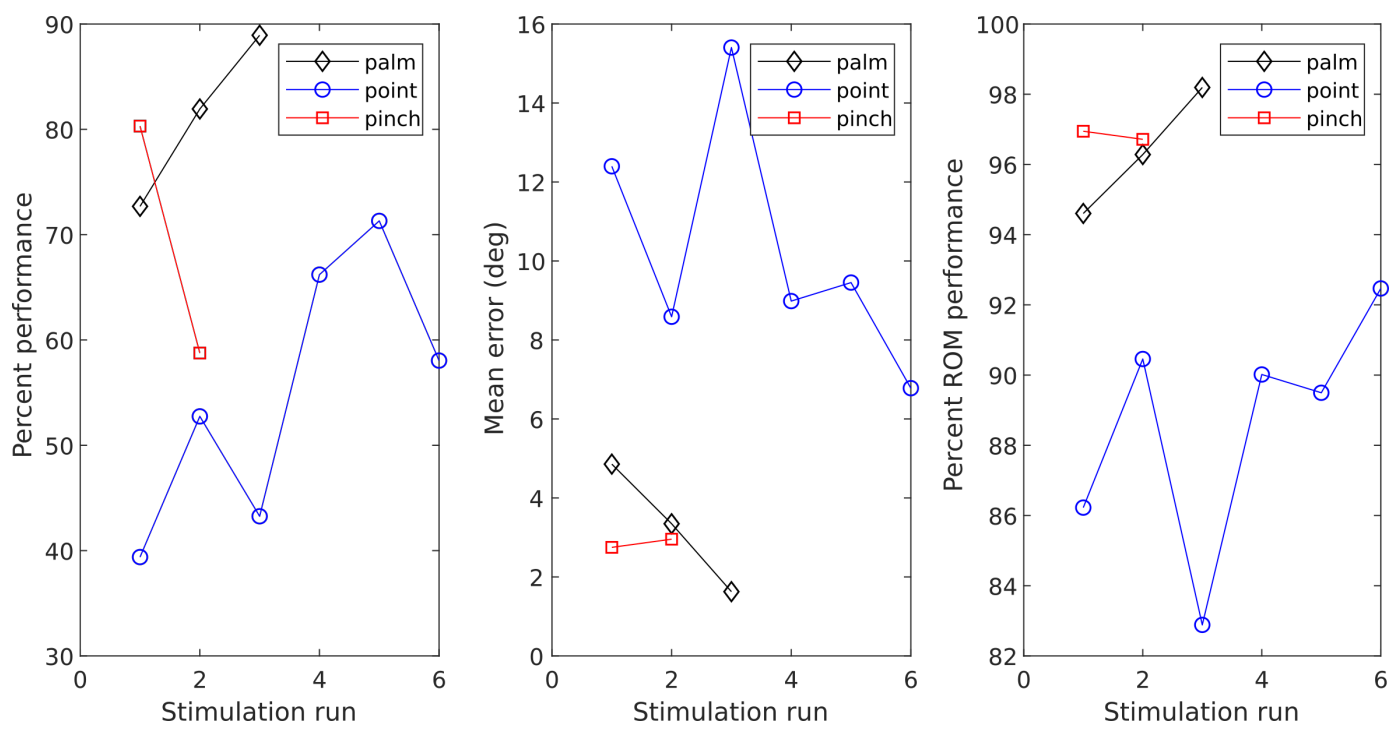

Figure 10. Graph showing reduction in error by iterative improvement of stimulation profile for the three different gestures.

The iterative learning algorithm altered the stimulation in subsequent stimulation runs which is reflected in the reduction in error in the centre graph as the number of stimulation runs increases. Figure 11 shows the mean error and percent performance recorded from the last run of each gesture on the five healthy participants using the same equations as in Figure 10. The performance exceeded $91 \%$ for all gestures and participants.
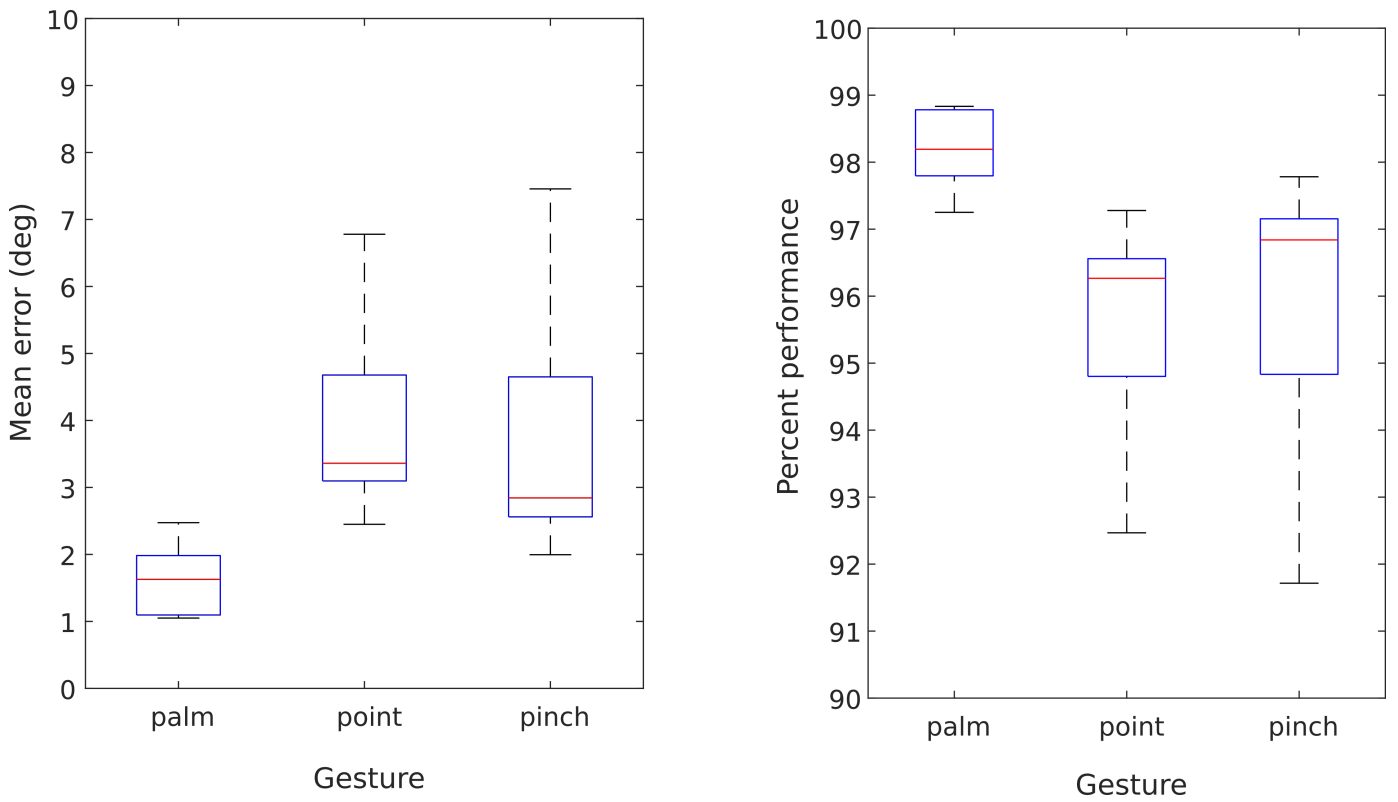

Figure 11. Graph comparing gesture performance on final run across participants. 


\subsubsection{Tests of Different Waveforms with Healthy Participants}

Tests with different waveform patterns were carried out on the healthy participants. Across the test group participants found that changing the stimulation waveform changed the amount of movement produced. Different participants, however, found different waveforms produced stronger results in their case. For example, one participant found the biphasic negative waveform produced the strongest results, for another the biphasic alternating waveform produced stronger results. This indicates that there are benefits to selecting the waveform on a per user basis. Further research will be required to investigate the best use of differing waveform patterns.

\subsubsection{Tests of Additional Upper Arm Stimulation with Healthy Participants}

The stimulation unit has also been tested with additional electrodes allowing stimulation of the upper arm to produce elbow extension movement. The new electrode was produced using the same dry electrode materials as the array, but has two large electrode pads each $5 \mathrm{~cm} \times 5 \mathrm{~cm}$, one for stimulation and one as an additional return. To connect this, one of the lower arm array electrodes was disconnected and its stimulation channel used for the new upper arm electrode. The same stimulation pulses were used for the upper arm electrode as was used on the electrodes on the wrist array. With this configuration, the system was able to produce contraction in the triceps muscles in addition to stimulating extension movements in the wrist and fingers. The use of additional electrodes on the upper arm therefore offers the option to create more complex actions using both hand and arm movements.

\subsubsection{Tests with Stroke Survivors}

Initial testing of the design on the wrist (i.e., lower arm only) with two of the EUG participants produced reduced movement when compared to identical tests with healthy users. The array identification phase of the test only produced limited movement, which resulted in very poor gesture stimulation.

There was almost no sensation before the unit reached its software defined pulse length limit indicating that the maximum stimulation level was too low. Tests varying the stimulation voltage used caused no obvious difference to either the comfort or movement generated by the system. To overcome this, the software was altered to increase the maximum pulse length to $1000 \mu$ s from the previously used level of $500 \mu \mathrm{s}$.

Another set of tests with the increased pulse length were carried out with two EUG members, one of whom was also in the previous group. These tests showed increased movement when compared with the previous tests. The first participant had a good level of movement with the system but wrist and finger extension was limited either by increased spasticity or by possible changes in reciprocal inhibition, whereby muscles on one side of a joint relax to accommodate contraction on the other side of the joint. After several tests the idle position of the wrist and fingers was more extended and relaxed. Due to this, there was a smaller range of movement observed from the stimulation as the starting point was closer to the maximum extension achievable. The idle position of the wrist and fingers returned back to a more flexed posture after a short period of time. The other participant had more movement than in their previous tests, however, additional movement would be required to closely achieve the target gestures. Despite this the wrist and fingers were again in a more relaxed position after the tests than before using the system. This needs further investigation and further tests such as using different stimulation waveforms are planned to optimize movement for the stroke survivors.

\section{Conclusions}

The new design for a multichannel biphasic stimulator discussed in this paper unlocks the possibility of achieving compact and low cost multichannel stimulators which are suitable for wearable 
deployment. In addition, tests show the device to be capable of generating the desired specificity and control over stimulation allowing complex multiple muscle gestures to be produced.

The use of an integrated high voltage driver simplifies and reduces the size of the electronics compared to designs using discrete switching components. Only nine additional components are required per channel using only $80 \mathrm{~mm}^{2}$ of PCB surface area in total. This means the additional circuitry for an eight channel driver fits in a $16 \mathrm{~mm}$ by $20 \mathrm{~mm}$ section of double sided PCB. In total, once the driver IC and routing space to link the parts together are taken into account an entire eight channel driver stage can be implemented in an area of $20 \mathrm{~mm}$ by $35 \mathrm{~mm}$ of 4-layer PCB space. The low cost per channel of the drivers makes large channel count stimulators feasible in terms of component and material cost.

When tested on healthy users the stimulator design, in conjunction with the rest of the SMARTmove system, is capable of creating predictable, controlled, and selective movements. Tests on stroke survivors have produced mixed results, some subjects have been limited by either spasticity in the flexor muscles, or possibly changes in the reciprocal inhibition patterns, whereas others have shown less response to the stimulator and are expected to need a stronger level of stimulation or a different waveform to achieve sufficient movement to realise the target gestures. Refinements to the system are being investigated with the aim of increasing the movement in those who had a weaker response to the stimulation provided.

Author Contributions: Conceptualization, C.F., J.T. and K.Y.; methodology, T.W., C.F., A.-M.H., J.T. and K.Y.; software, T.W., C.F.; validation, C.F.; formal analysis, C.F.; investigation, T.W., A.-M.H. and C.P.; resources, N.G., Y.W. and K.Y.; data curation, C.F.; writing—original draft preparation, T.W.; writing-review and editing, T.W., J.T. and K.Y.; visualization, C.F.; supervision, K.Y.; project administration, K.Y.; funding acquisition, C.F., J.T. and K.Y. All authors have read and agreed to the published version of the manuscript.

Funding: This research was funded by Medical Research Council (grant ref: MR/N027841/1) and EPSRC (grant ref: EP/S001654/1).

Acknowledgments: We would like to acknowledge the support of the SMART-move project End User Group participants and Advisory Board Members. Ethical approval was received from the Faculty of Health Sciences Ethics Committee, University of Southampton, reference 23824.A7.

Conflicts of Interest: K.Y. and J.T. are co-founders of Smart Fabric Inks Ltd which produced the inks used on the fabric electrodes.

\section{References}

1. Institute for Metrics and Health Evaluation. Global Burden of Disease Tool. 2017. Available online: http:/ / ghdx.healthdata.org/gbd-results-tool (accessed on 25 May 2020).

2. Royal College of Physicians. National Clinical Guideline for Stroke. Available online: https:/ /www.strokeaudit. org/SupportFiles/Documents / Guidelines / 2016-National-Clinical-Guideline-for-Stroke-5t-(1).aspx (accessed on 25 May 2020).

3. Pollock, A.; Baer, G.; Campbell, P.; Choo, P.L.; Forster, A.; Morris, J.; Pomeroy, V.M.; Langhorne, P. Physical rehabilitation approaches for the recovery of function and mobility following stroke. Cochrane Database Syst. Rev. 2014, 9, 965-967. [CrossRef] [PubMed]

4. Vafadar, A.K.; Côté, J.N.; Archambault, P.S. Effectiveness of functional electrical stimulation in improving clinical outcomes in the upper arm following stroke: A systematic review and meta-analysis. BioMed Res. Int. 2015, 2015, 1-14. [CrossRef] [PubMed]

5. Teasell, R.W.; Foley, N.C.; Bhogal, S.K.; Speechley, M.R. An evidence-based review of stroke rehabilitation. Top. Stroke Rehabil. 2003, 10, 29-58. [CrossRef] [PubMed]

6. SMART Move Website. Available online: www.smartmove.soton.ac.uk/ (accessed on 20 February 2020).

7. Popović-Maneski, L.; Topalović, I. EMG Map for Designing the Electrode Shape for Functional Electrical Therapy of Upper Extremities. In Converging Clinical and Engineering Research on Neurorehabilitation III, Proceedings of the 4th International Conference on NeuroRehabilitation (ICNR2018), Pisa, Italy, 16-20 October 2018; Springer: Berlin/Heidelberg, Germany, 2018; pp. 1003-1007. [CrossRef] 
8. Kuhn, A.; Keller, T.; Micera, S.; Morari, M. Array electrode design for transcutaneous electrical stimulation: A simulation study. Med. Eng. Phys. 2009, 31, 945-951. [CrossRef] [PubMed]

9. Crema, A.; Furfaro, I.; Raschellà, F.; Micera, S. Development of a hand neuroprosthesis for grasp rehabilitation after stroke: State of art and perspectives. In Converging Clinical and Engineering Research on Neurorehabilitation III, Proceedings of the 4th International Conference on NeuroRehabilitation (ICNR2018), Pisa, Italy, 16-20 October 2018; Springer: Berlin/Heidelberg, Germany, 2018; pp. 89-93. [CrossRef]

10. Kenney, L.P.; Heller, B.W.; Barker, A.T.; Reeves, M.L.; Healey, J.; Good, T.R.; Cooper, G.; Sha, N.; Prenton, S.; Liu, A.; et al. A review of the design and clinical evaluation of the ShefStim array-based functional electrical stimulation system. Med. Eng. Phys. 2016, 38, 1159-1165. [CrossRef] [PubMed]

11. Velik, R.; Malešević, N.; Maneski, L.; Hoffmann, U.; Keller, T. INTFES: A Multi-pad Electrode System for Selective Transcutaneous Electrical Muscle Stimulation. In Proceedings of the 16th Annual International Functional Electrical Stimulation Society Conference, São Paulo, Brazil, 8-11 September 2011.

12. Heller, B.W.; Clarke, A.J.; Good, T.R.; Healey, T.J.; Nair, S.; Pratt, E.J.; Reeves, M.L.; van der Meulen, J.M.; Barker, A.T. Automated setup of functional electrical stimulation for drop foot using a novel 64 channel prototype stimulator and electrode array: Results from a gait-lab based study. Med. Eng. Phys. 2013, 35, 74-81. [CrossRef] [PubMed]

13. Souza, D.C.D.; Gaiotto, M.D.C.; Nogueira Neto, G.N.; Castro, M.C.F.D.; Nohama, P. Power amplifier circuits for functional electrical stimulation systems. Res. Biomed. Eng. 2017, 33, 144-155. [CrossRef]

14. Popovic, M.; Curt, A.; Keller, T.; Dietz, V. Functional electrical stimulation for grasping and walking: Indications and limitations. Spinal Cord 2001, 39, 403-412. [CrossRef] [PubMed]

15. Huerta, S.C.; Tarulli, M.; Prodic, A.; Popovic, M.R.; Lehn, P.W. A universal functional electrical stimulator based on merged flyback-SC circuit. In Proceedings of the 2012 15th International Power Electronics and Motion Control Conference (EPE/PEMC), Novi Sad, Serbia, 4-6 September 2012; pp. LS5a.3-1-LS5a.3-5. [CrossRef]

16. Freeman, C.; Spraggs, M.W.; Hughes, A.M.; Yang, K.; Tudor, M.; Grabham, N. Multiple model adaptive ILC for human movement assistance. In Proceedings of the IFAC European Control Conference 2018, Limassol, Cyprus, 12-15 June 2018; pp. 1-6. [CrossRef]

17. Yang, K.; Meadmore, K.; Freeman, C.; Grabham, N.; Hughes, A.M.; Wei, Y.; Torah, R.; Glanc-Gostkiewicz, M.; Beeby, S.; Tudor, M. Wearable electronic sleeve for muscle stimulation. In Proceedings of the Annual Conference of the International Functional Electrical Stimulation Society 2018, Nottwil, Switzerland, 28-31 August 2018.

(C) 2020 by the authors. Licensee MDPI, Basel, Switzerland. This article is an open access article distributed under the terms and conditions of the Creative Commons Attribution (CC BY) license (http://creativecommons.org/licenses/by/4.0/). 